Ajda Gabrič

ajda.gabric@gmail.com
UDK 821.112.2.09-93"19/20":81'42

DOI: $10.4312 /$ vestnik.8.153-168

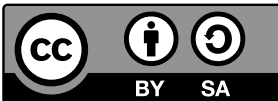

\title{
INTERTEXTUALITÄT BEI MICHAEL ENDE, CORNELIA FUNKE UND WALTER MOERS
}

\section{$1 \quad$ EINLEITUNG}

Der Beitrag setzt sich mit den intertextuellen Bezügen in drei literarischen Texten auseinander. Analysiert werden Romane, die in der Regel als phantastische Romane für Kinder bzw. Jugendliche gelten:

Michael Endes Die unendliche Geschichte (1979) berichtet vom Schüler Bastian, der durch das Lesen des Buches „Die unendliche Geschichte“ ins wundervolle Land Phantásien reist. Am Ende findet er den Weg zurück in seine eigene Welt.

Cornelia Funkes Tintenherz (2003) erzählt vom Buchbinder Mo, der Figuren aus Geschichten „herauslesen“ kann, so dass sie plötzlich körperlich vor ihm stehen. Diese Fähigkeit löste in der Vorgeschichte die ganze Handlung des Romans aus, als er drei Charaktere aus dem Buch ,Tintenherz“ holte. Jahre später muss er deswegen seine Tochter Meggie und sich selber bei der exzentrischen Tante Elinor verstecken. Nach einer Reihe von Abenteuern erwartet sie alle ein glückliches Ende.

Walter Moers' Die Stadt der Träumenden Bücher (2004) ist eine fiktive Autobiografie. Der Protagonist ist der Lindwurm Hildegunst, der nach dem Tod seines Paten Danzelot in dessen Nachlass eine Handschrift findet und sich entscheidet, sich auf die Suche nach deren Autor zu begeben. Er wird vom bösen Phistomefel überlistet, verläuft sich in den Labyrinthen unterhalb der Stadt, lernt vom mysteriösen ,Schattenkönig“ über Literaturschreiben und findet am Ende wieder auf die Oberfläche zurück.

Den Romanen ist neben ihrer phantastischen Handlung gemeinsam, dass in ihnen viele Zitate aus anderen literarischen Texten und Anspielungen auf diese vorkommen - die Romane weisen einen hohen Grad an Intertextualität auf. Es wird im Beitrag analysiert, aus welchen Texten die intertextuellen Bezüge überwiegend stammen, wie explizit sie sind und welche Funktion sie innerhalb des neuen Textes übernehmen. Die Analyse lehnt sich vor allem an den Theorieansatz von Gérard Genette an, der Intertextualität als eine der fünf Arten der sogenannten Transtextualität definiert. 


\section{2 KONZEPT DER INTERTEXTUALITÄT}

Das zentrale Konzept dieses Artikels ist die Intertextualität als definierende Eigenschaft der analysierten Romane. Ihre breiteste Definition kann schon dem Metzler Lexikon Literatur- und Kulturtheorie entnommen werden: Intertextualität soll dem zufolge „die Eigenschaft von insbes. literar. Texten, auf andere Texte bezogen zu sein“, bedeuten, wobei die Intertextualitätstheorien die Bezüge zwischen Texten beschreiben, erklären oder systematisieren (Nünning 2013: 349). Der Begriff entwickelte sich in den sechziger Jahren im Kontakt zwischen dem Osten und dem Westen in einer interdisziplinären Interaktion der Literaturwissenschaft, Semiotik, Linguistik, Psychoanalyse, Mathematik, Logik und Philosophie (Juvan 2000: 5).

Die Intertextualität wurde bei Julia Kristeva, die den Ausdruck einführte, als eine Eigenschaft aller Texte verstanden (Nünning 2013: 349). Es handelte sich dabei um die Verbindungen aller Texte, die in Beziehungen zueinander standen. Später entwickelten sich die Intertextualitätstheorien in zwei verschiedene Richtungen. Es geht um den Unterschied zwischen Theorien, ,die davon ausgehen, dass Intertextualität im literarischen Text lokalisiert und markiert werden muss, um theorierelevant zu werden, und solchen, die Intertextualität als Seinsbedingung von Kultur bestimmen (Berndt/Tonger-Erk 2013: 12). Die Theorien, die sich an der markierten Intertextualität der Texte orientieren, sind für die Anwendung bei der Analyse spezifischer literarischer Texte deutlich brauchbarer, deshalb wurde die Definition Genettes ausgewählt, um die Romane zu beschreiben.

Gérard Genette orientiert sich an den „deskriptiven“ Theorien der Intertextualität, da bei ihm nur die direkten Bezüge auf andere literarische Werke innerhalb später entstandener literarischer Werke als intertextuelle Elemente gelten. Er definiert in seinem Buch Palimpseste: Die Literatur auf zweiter Stufe die Intertextualität mit den vier anderen Formen dessen, was er Transtextualität oder textuelle Transzendenz des Textes nennt. Die Transtextualität ist bei ihm ,alles das [...], was ihn [den Text] in eine manifeste oder geheime Beziehung zu anderen Texten bringt" (Genette 1993: 9). Den breiteren Begriff der Intertextualität, wie ihn Kristeva zunächst definierte, ersetzt er also mit Transtextualität, wobei die Intertextualität viel enger und spezifischer aufgefasst wird.

Sie ist die erste Form der Transtextualität und wird folgendermaßen definiert: „Beziehung der Kopräsenz zweier oder mehrerer Texte, d. h. in den meisten Fällen, eidetisch gesprochen, als effektive Präsenz eines Textes in einem anderen Text." (Genette 1993: 10) Einfacher gesagt, ein Text ist in einem anderen Text präsent, und zwar auf verschiedene Weisen: Das Zitat soll die einfachste und wörtlichste Form der Intertextualität sein (ebd., 10), die zweite Form der Intertextualität ist das Plagiat, die „eine nicht deklarierte, aber immer noch wörtliche Entlehnung darstellt“ (ebd., 10). Die dritte Form der intertextuellen Beziehungen ist die Anspielung, die weniger explizit und weniger wörtlich ist als das Zitat und das Plagiat; sie ist die Aussage, deren Verständnis „,das Erkennen einer Beziehung zwischen ihr und einer anderen voraussetzt“ (ebd., 10). Frauke 
und Tonger-Erk weisen auf die nicht völlig ausgearbeitete Unterscheidung zwischen einem Plagiat und einem Zitat hin; sie sind an der bloßen Oberfläche des Textes nicht zu unterscheiden (Frauke/Tonger-Erk 2013: 116). Der Unterschied sei nur vom Kontext erkennbar. Bei der Analyse der behandelten Romane wird nur von Zitaten gesprochen. Die intertextuellen Verfahren sind darin nämlich spielerisch eingesetzt und so in einen neuen Kontext gestellt, dass eher von Zitaten als von Plagiaten zu sprechen ist. Die unendliche Geschichte enthält meistens undentifizierte Zitate, verändert aber ihren Kontext und ihre Stellung im literarischen Text so, dass sie eine völlig neue Bedeutung bekommen. Bei Die Stadt der Träumenden Bücher wird meistens versteckt darauf hingewiesen, dass es sich um die Arbeit eines anderen Autors handelt. Tintenherz enthält dagegen ein Quellenverzeichnis, worin alle ursprünglichen Texte, aus denen Zitate und Anspielungen stammen, identifiziert werden.

Neben der Intertextualität führt Genette noch weitere Formen der transtextuellen Bezüge ein. Die Paratextualität ist die Beziehung, die der Text mit seinem Paratext bildet. Unter Paratext sind alle „Arten zusätzlicher, auto- oder allographer Signale, die den Text mit einer (variablen) Umgebung ausstatten und manchmal mit einem offiziellen oder offiziösen Kommentar versehen" (Genette 1993: 11), zu verstehen. Es geht um die Beziehung des Textes zu seinen Titeln, Untertiteln, Vorworten, Nachworten, Hinweisen an den Leser, Einleitungen usw. Der dritte Typ der Transtextualität heißt Metatextualität und bezieht sich auf das Verhältnis zwischen einem Text und der kritischen Auseinandersetzung mit diesem Text. Die Hypertextualität bezeichnet ,jede Beziehung zwischen einem Text B (den ich als Hypertext bezeichne) und einem Text A (den ich, wie zu erwarten, als $H y$ potext bezeichne), wobei Text B Text A auf eine Art und Weise überlagert, die nicht die des Kommentars ist." (ebd., 14-15) Der zweite Text wird als eine Ableitung des ersten gesehen. Der letzte Typ ist die Architextualität und bezeichnet die Zugehörigkeit eines Textes zu einer Gattung oder überhaupt zur Literatur. Diese ist abstrakt und implizit und bleibt meistens unausgesprochen oder wird seltener in einem paratextuellen Hinweis wie „Roman“ oder „Erzählung“ expliziert (Genette 1993: 10, 13).

Die drei Romane werden mithilfe von Genettes Theoriebegriffen analysiert, und zwar werden die Ausführungen auf die Intertextualität beschränkt - wichtig ist dabei die Unterscheidung zwischen den Zitaten und Anspielungen. Auch die paratextuellen Elemente sind für das Verständnis der behandelten Romane von Bedeutung; eine nähere Auseinandersetzung damit würde aber den Rahmen dieses Artikels sprengen. Die Begriffe der Meta-, Hyper- und Architextualität sind für die Analyse dieser Texte weniger relevant. 


\section{INTERTEXTUALITÄT IN ROMANEN}

\subsection{Mehr oder weniger wörtliche Zitate}

In den analysierten Texten gibt es eine Menge von Zitaten aus anderen literarischen oder auch einigen nichtliterarischen Texten und Anspielungen an andere Texte. Sie erfüllen in den drei Romanen verschiedene Funktionen.

Tintenherz enthält besonders viele Zitate aus Texten, die auch für Kinder geeignet oder sogar für Kinder geschrieben worden sind, z. B. James Barries Peter Pan oder Roald Dahls Hexen Hexen. Jedes Kapitel fängt mit einem Zitat aus einem anderen, meistens literarischen, Text an. Unter den Zitaten stehen der Name ihres Autors und der Titel des Textes, aus dem sie stammen, und am Ende des Bandes steht auch ein Quellenverzeichnis. Dies ist für ein literarisches Werk unüblich, da die Zitate darin nicht notwendig (eindeutig) identifiziert werden müssen, wie es zum Beispiel für Die unendliche Geschichte und Die Stadt der Träumenden Bücher gilt. Die Anführung der Quellen hat mit der belehrenden und erzieherischen Funktion des Buches als Kinderbuch zu tun. Auf diese Weise können die jungen Leser ihren Lesehorizont erweitern oder aber die Texte wiedererkennen, die sie schon gelesen haben. Die Mottos haben eine zweifache Funktion: Einerseits thematisieren sie die Literatur oder das Erzählen selbst und andererseits leiten sie das Thema oder die Handlung des folgenden Kapitels ein. Zweiundzwanzig von neunundfünfzig Mottos zu den Kapiteln beschäftigen sich mit dem Konzept der Selbstreferenz, also thematisieren sie die Literatur innerhalb eines literarischen Textes, und zwar durch das Thematisieren des Lesens oder ähnlicher Phänomene oder durch die Wörter wie „Buch“ oder „Lied“ (Heber 2010: 30). Die meisten Zitate sagen kurz den Inhalt des Kapitels voraus oder fassen ihn zusammen, fungieren also als eine Art Einleitung und Anweisung zum Lesen des Kapitels. Als Beispiel kann die Einleitung ins Kapitel „Der erfüllte Auftrag" angeführt werden:

„Es hat keinen Zweck, ihn zu suchen“, knurrte der Biber.

„Was soll das heißen?“, fragte Suse. „Er kann doch nicht weit sein! Wir müssen ihn finden! Warum behaupten Sie, dass es keinen Zweck hat, ihn zu suchen?“ „Weil es ganz klar ist, wo er ist“, antwortete der Biber. „Begreift ihr denn nicht? Er ist zu ihr gegangen, zur Weißen Hexe. Und er hat uns verraten!

C. S. Lewis, Der König von Narnia (Funke 2014: 137)

Es handelt sich um ein Zitat aus Der König von Narnia, dem zweiten Band von Die Chroniken von Narnia von Clive S. Lewis. Im Abschnitt geht es darum, dass die Protagonisten gerade begriffen haben, dass ihr Bruder nicht mehr da ist, und der Biber erklärt ihnen, dass er sie alle verraten hat. Im folgenden Kapitel von Tintenherz wird eine ähnliche Situation beschrieben: Aus den Worten „Das Mädchen und das Buch, habe ich 
gesagt“ (Funke 2014: 141), die Capricorn an Staubfinger richtet, erkennt Meggie, dass Staubfinger für Capricorn arbeitete und dass er sie, ihren Vater und Tante Elinor verriet. Das Zitat und das Kapitel sind also durch die Beschreibung einer ähnlichen Situation, Verrat der Protagonisten und Protagonistinnen durch jemanden, dem sie vertraut hatten, verbunden. Andere Zitate haben meistens dieselbe Funktion, eine ähnliche Situation, wie das folgende Kapitel sie bietet, zu zeichnen, sie können aber auch die literarischen Texte einleiten, die im Kapitel für die Handlung wichtig werden. Vor dem Kapitel „Zauberzunge" (ebd., 191), in dem Mo aus Stevensons Die Schatzinsel und aus Tausend und eine Nacht für Capricorn Schätze herauslesen muss, steht so ein Zitat aus Die Schatzinsel.

Neben den Einleitungen zu den einzelnen Kapiteln kommen Zitate auch im Kerntext vor. Sie sind „für den Rezipienten sofort wegen des Kursivdruckes erkennbar“ (Heber 2010: 100). Sie erfüllen zweierlei Funktionen: Einerseits dienen sie der Charakterisierung der Figuren und andererseits treiben sie die Handlung voran. Sie sind Teil der Kommunikation zwischen Figuren und nicht Teil der Erzählerrede. Die Figuren, die auswendig aus anderen literarischen Texten zitieren, sind vor allem Fenoglio und Elinor, die dadurch ihre Belesenheit zeigen, zum Beispiel, wenn Fenoglio über den Räuber Basta spricht: „Wie heißt es so schön in einem wunderbaren Buch? Es ist schrecklich leicht, Kinder davon zu überzeugen, dass sie abscheulich sind. Basta war überzeugt davon.“ (Funke 2014: 362) Fenoglio charakterisiert in diesen Worten, die durch den vorhergehenden Satz und Kursivdruck explizit als Zitat identifiziert werden, sich selber als einen belesenen Mann und zugleich Basta als einen Menschen, der als Kind keine Liebe erfahren hatte. Von Heber (2010: 105) wird Dickens' Oliver Twist als Quelle des Zitats identifiziert, was zeigt, dass Fenoglio über Wissen über klassische Literatur verfügt. Die meisten Zitate erfüllen so die Rolle der Vertiefung der Charakterisierung. Die andere Funktion, das Vorantreiben der Handlung, wird vor allem in den Fällen wichtig, wo entweder Mo oder Meggie etwas oder jemanden aus den literarischen Texten herauslesen, was später die Handlung lenkt. Meggie liest zum Beispiel aus Peter Pan und plötzlich sieht sie ein Licht im Zimmer, „wie es hin und her schwirrte, hastig, schneller als ein Glühwürmchen und viel größer.“ (Funke 2014: 388) Das Licht erweist sich als die Fee Tinker Bell und ihre Präsenz im Zimmer lässt Capricorns Räuber wissen, dass auch Meggie Mos Gabe hat und Gegenstände und Lebewesen aus Büchern herauslesen kann. Das Zitieren der Texte in den Fällen, wo Meggie oder Mo laut vorlesen, hat also eine Funktion auf der Ebene der Handlung.

Heber hebt hervor, dass Intertextualität nur ein „Bezug eines literarischen Werkes auf andere, real existente Primärwerke oder auf tradierte und im kulturellen Gedächtnis gespeicherte Stoffe“ (Heber 2010: 19) ist; Intertextualität sei „an die reale Existenz eines Werkes gebunden“ (ebd., 20). Dies schließt die Textausschnitte aus, die andere fiktive Texte anführen, z. B. Fenoglios „Tintenherz“ und seinen zusätzlichen Text, mit dem er die Geschichte von „Tintenherz“ zu ändern versucht. In solchen Beispielen sei „,bestenfalls Intertextualität vorgetäuscht oder ist fiktiv" (ebd., 20).

In Die Stadt der Träumenden Bücher kommen genauso Zitate vor, sie werden aber im Unterschied zu Tintenherz nicht genau den Autoren und primären Texten zugeschrieben, 
von denen sie stammen. Moers hat eine besondere Art, mit Zitaten und überhaupt mit intertextuellen Bezügen umzugehen. Es kommt im Roman eine Vielzahl von Zitaten aus anderen literarischen Werken vor, die entweder aus realen literarischen Werken, aus der Literaturgeschichte bekannt, oder von den von ihm erfundenen ,zamonischen“ Autoren stammen $^{1}$ - was die von Heber thematisierte fiktive oder vorgetäuschte Intertextualität ist. Die Aufgabe, zu entziffern, ob es sich um ein Zitat aus einem realen literarischen Werk handelt oder nicht, wird den Rezipierenden überlassen, die aber vom Text Hinweise für die Lesart bekommen, und zwar sind ein Großteil der Namen von ,zamonischen“ Autoren eigentlich Anagramme der Namen realer Schriftsteller/-innen und Dichter/-innen. Diese verlangen von Lesenden ein Vorwissen über die deutschsprachige und europäische Literaturgeschichte und eine besondere Aufmerksamkeit bei der Rezeption. Die Zitate sind entweder wortwörtlich übernommen oder etwas verändert, jedoch nicht genug, als dass die Verbindung zum Original verloren ginge. Als Beispiel eines Zitats, das teilweise verändert wurde, jedoch noch deutlich erkennbar für die meisten Leser, die sich in der deutschsprachigen Literaturgeschichte auskennen, kann das Gedicht „Ojahnn Golgo van Fonthewegs" Nurnenwald angeführt werden:

\author{
Über allen Gipfeln \\ Ist Ruh, \\ In allen Wipfeln \\ Spürest du \\ Kaum einen Hauch \\ Die Nurnen schweigen im Walde \\ Warte nur, balde \\ Ruhest du auch.
}

(Moers 2015: 246)

Hinter dem Namen Ojahnn Golgo van Fontheweg steckt deutlich eine Anspielung an Johann Wolfgang von Goethe und das zitierte Gedicht ist eigentlich Goethes Gedicht Wanderers Nachtlied; der einzige Unterschied zwischen Goethes und „Fonthewegs“ Gedicht ist, dass das Wort „Vögelein“ mit dem Wort „Nurnen“ ausgetauscht worden ist (vgl. Goethe 1987: 53). Dies verleiht dem Gedicht eine „,zamonischere“ Note, da Nurnen eine phantastische, von Moers erfundene Tierart aus Zamonien sind. Sie sollten ein äuBerst gefährliches „Amalgam des Grauens aus totem Laub, Blut und Leichen“(Dollinger/ Moers 2012: 182) sein, was dem Gedicht eine besonders unheimliche Note verleiht.

Die andere Art „Zitate“ sind die, die eigentlich von keinem anderen Text stammen, von dem Erzähler aber anderen, nicht realen Autoren zugeschrieben werden, wie zum Beispiel Hildegunsts Paten Danzelot. Sein Name ist kein Anagramm eines realen

1 Die Handlung des Romans findet im fiktiven Land Zamonien statt. 
Schriftstellers und sein Gedicht (Moers 2015: 22) ist kein Zitat aus den Werken eines anderen Dichters, also kein Zeichen der Intertextualität im Text (Heber 2010: 19-21). Die Zitate müssen von Leser oder Leserin selber als fiktives Zitieren gegenüber den Zitaten aus realen literarischen Werken erkannt werden. Auf diese Weise wird der Prozess der Rezeption verzögert, da eine gewisse Aufmerksamkeit bei der Lektüre vorausgesetzt wird. Neben der Verzögerung des Rezeptionsprozesses bringen Zitate auch einen humorvollen Ton in den Text. Das Spielerische wird dadurch eingesetzt, dass in die kanonischen Texte plötzlich absurde phantastische Wesen wie Nurnen ihren Weg finden. Das spielerische Ersetzen der Wörter in klassischen Texten wirkt also humorvoll und bringt eine Wiederbelebung der klassischen Autoren mit sich.

In Die unendliche Geschichte sind die Zitate ebenso präsent, es gibt aber weniger von ihnen und sie sind nicht so offensichtlich als Zitate markiert wie in den beiden anderen Romanen. Sie passen sich vielmehr dem neuen Kontext an. Eine Stelle, wo im Kontext angedeutet wird, dass es sich bei einem Textteil um ein Zitat handelt, zitiert aus Shakespeare. Die drei Ritter, mit denen Bastian durch Phantásien reist, singen nämlich gerne ein Lied:
„Als ich ein kleines Büblein war, juppheißa bei Regen und Wind ..."“
Wie sie erklärten, stammte es von einem Phantásienreisenden aus längst vergangenen Tagen, der Schexpir oder so ähnlich geheißen hatte.

(Ende 2014: 321-33)

Es handelt sich um ein Zitat aus Shakespeares Komödie Was ihr wollt. ${ }^{2}$ Es wird angedeutet, dass Shakespeare so wie Bastian Phantásien als Inspirationsquelle ausgesucht haben soll. Ende verfährt in diesem Fall ähnlich wie Moers mit seinen Anagrammen - der Name des realen Autors wird teilweise verkehrt - bei Moers anagrammiert, bei Ende falsch geschrieben, der reale Name bleibt aber immer noch erkennbar. Das andere augenfällige Zitat ist das Motto Phantásiens, das auf dem magischen Symbol AURYN steht, ,das Zeichen dessen, der im Auftrag der Kindlichen Kaiserin stand und in ihrem Namen handeln konnte, so als sei sie selbst anwesend.“ (Ende 2014: 46) Das Amulett bekommt Bastian von der Kaiserin bei seinem Eintritt in Phantásien und bemerkt, dass auf seiner Rückseite die Inschrift „Tu Was Du Willst“" (ebd., 237) steht. Es handelt sich um ein Zitat aus François Rabelais' Roman Gargantua und Pantagruel, worauf aber in Die unendliche Geschichte nie hingedeutet wird. Im Originalkontext bedeutet der Satz, der sich auf die Regel des Lebens in einer Abtei bezieht, dass man nach seiner eigenen Willkür immer das tun darf, was einem beliebt (vgl. Rabelais 1981: 242). Das Zitat hat sich ganz dem neuen Kontext angepasst und bedeutet jetzt genau das Gegenteil davon, und zwar, dass Bastian seinen

2 Originaltitel Twelfth Night, or, What You Will, die zitierte Stelle befindet sich in Shakespeare 2001: 104. 
wahren Willen finden muss, um Phantásien produktiv gestalten zu können und schließlich auch wieder nach Hause zu finden. Es geht nicht um beliebige Willkür, sondern um einen innerlichen Trieb, der Folgen für die ganze Menschheit haben kann und womit folglich vorsichtig umgegangen werden muss.

\subsection{Verstecktere Anspielungen}

Neben direkten Zitaten findet man in allen drei Romanen auch indirekte Anspielungen an andere konkrete literarische Texte, Genres, historische Stile oder Traditionen. Sie erfüllen teilweise dieselben Funktionen wie die direkten Zitate, sind aber in der Regel weniger offensichtlich und schwieriger zu identifizieren.

Die Anspielungen in Tintenherz sind meistens als solche erklärt, was nicht mit Genettes Meinung übereinstimmt, dass Anspielungen wenig explizit sind - zum Beispiel schreiben sich Mo und Meggie Nachrichten in einer Schrift, die Elinor und Fenoglio nicht lesen könnnen, und Mo und Meggie erklären ihnen, dass sie aus Tolkiens Der Herr der Ringe stammt (Funke 2014: 425). Heber (2010: 82-100) identifiziert fast alle Quellen der Anspielungen und es ist bei ihnen, so wie bei den direkten Zitaten, augenfällig, dass sie meistens aus Texten stammen, die entweder für Kinder geschrieben wurden oder auch Kindern oder Jugendlichen zugänglich sind. Auch die erwachsenen Figuren Mo und Elinor sprechen öfter von Texten, die auch die zwölfjährige Meggie verstehen kann, als von Texten, die ausschließlich Erwachsenen zugänglich sind. Dies hat die Aufgabe, sich den kindlichen Lesern und Leserinnen anzunähern, damit sie sich leichter mit den Figuren identifizieren und sich im Roman erkennen. Es wirkt gleichzeitig als eine Empfehlung zur Lektüre dieser literarischen Texte - der Roman bekommt dadurch wieder eine didaktische Note. Die Anspielungen sind wahrscheinlich aus demselben Grund oft auch explizit durch den Titel identifiziert: Es handelt sich um ein Buch für Kinder, das wegen seines Zielpublikums einfacher als die Bücher für Erwachsene sein muss, um verständlich zu sein, und das seine Leser vielleicht auch belehren will. Den zweiten Grund für die Deutlichkeit der Anspielungen sehe ich in der Tatsache, dass die Elemente aus den anderen literarischen Texten auch auf die Ebene der Handlung treten oder die Figuren näher charakterisieren und nicht nur eine zusätzliche Kuriosität auf der Ebene des Diskurses darstellen, deshalb müssen sie explizit sein, um verständlich für die jüngeren Leser zu werden. Tante Elinor wird z. B. durch die Hinzuziehung einer Anspielung näher vorgestellt:

„Elinor ist sehr stolz auf dieses Tor. Sie hat es eigens anfertigen lassen, nach einem Bild in einem Buch.“

„Ein Bild vom Garten des selbstsüchtigen Riesen?“, murmelte Meggie, während sie durch die kunstvoll verschlungenen Eisenstäbe lugte.

„Der selbstsüchtige Riese?“ Mo lachte. „Nein, ich glaube, es war eine andere Geschichte. Obwohl die sehr gut zu Elinor passen würde.“ (Funke 2014: 40) 
Es handelt sich um eine Anspielung auf Oscar Wildes Erzählung Der selbstsüchtige Riese (identifiziert von Heber 2010: 94-95) und durch den Vergleich zwischen Elinor und dem Riesen wird schon vor Elinors Auftritt eine Erwartung ihres Charakters gebildet - sie soll unfreundlich sein und vor allem keine Kinder mögen. Dies bestätigt sich, bald nachdem Meggie Tante Elinor kennenlernt.

Viele in Die Stadt der Träumenden Bücher erwähnte ,fiktive“ Autoren und eine Autorin sind Anspielungen auf reale Personen, die aus der überwiegend deutschsprachigen, teilweise aber auch europäischen Literaturgeschichte bekannt sind. Sie verstecken sich hinter den "teilweise sehr offensichtlichen Anagrammen und Werkzitaten“ (Conrad 2011: 290). Das offensichtlichste Beispiel ist meines Erachtens der schon erwähnte „Ojahnn Golgo van Fontheweg“, dessen Name ein Anagramm von Johann Wolfgang von Goethes Namen ist und der durch seine Offensichtlichkeit als Schlüssel zur Lektüre anderer Namen „zamonischer“ Schriftsteller fungiert. Die Grenze zwischen dem Zitieren und der Anspielung kann in diesem Roman nicht klar gezeichnet werden, da nicht klar ist, ob ein Zitat noch immer ein Zitat ist, wenn, wie im Nurnenwald, darin ein Wort vertauscht wird, oder ob es sich hier „nur" noch um eine Anspielung handelt. Die Namen der Autoren wären meiner Meinung nach schon eher eine Anspielung, da nicht immer offensichtlich ist, wer, wenn überhaupt jemand, sich hinter dem Anagramm versteckt und weil den ,zamonischen“ Autoren manchmal Eigenschaften zugeschrieben werden, die für die realen Autoren nicht gelten.

Einige von den Namen tragen sogar als Anagramme eine parodistische Abwertung der einzelnen Autoren bzw. beziehen sich auf dessen Werk:

Eduard Mörike wird in SdTB zu Akud Ödreimer, wobei deutlich wird, dass durch dieses Anagramm die mangelnde Spannung in seinen Werken ausgedrückt werden soll. Dölerich Hirnfidler ist die anagrammatische Entsprechung von Friedrich Hölderlin. Hirnfidler kann als Anspielung auf die (vermeintliche) Geisteskrankheit Hölderlins verstanden werden. Zuletzt wird Hugo Ball als Vertreter des Dadaismus zu Hulgo Bla als Vertreter des Gagaismus. Durch drei Elemente kommt es zu einer Parodierung Balls: durch den umgangssprachlichen Ausdruck ,bla ‘ für Sinnloses Gerede, durch die inhaltlichen Eigenheiten, die ihm zugeschrieben werden, und durch die Gattung, zu der seine Literatur zugeordnet wird, deren Bezeichnung abgeleitet vom Wort, gaga' bereits alles verrät[.] (Mader 2012: 170)

Der Nachname „Hirnfidler“ kann nicht nur als eine Anspielung auf seine Geisteskrankheit, sondern auch als eine Kritik der Melodie seiner Gedichte interpretiert werden - der Name impliziert, dass sich seine Texte so wie eine Geige im Hirn anhören. Meines Erachtens kann aber auch „T. T. Kreischwurst“ bzw. Kurt Schwitters (identifiziert von Conrad 2011: 290) zu dieser Gruppe gezählt werden, denn erstens impliziert der Name ähnlich wie bei „Hirnfidler“ etwas, was unangenehm ist zu hören, zweitens ist er aber völlig sinnlos, 
was einen Bezug zu seinem Stil des Dadaismus herstellt. Neben Anspielungen auf konkrete Autoren findet man im Roman auch Anspielungen auf Literaturgattungen oder literarische Stile. „Zamonischer Gagaismus“, für den Stottern, anscheinend sinnlose Gedichte und die Zusammenarbeit mehrerer Autoren in einer Gruppe typisch sein sollten (Moers 2015: 234), kann als eine Anspielung auf den modernistischen Stil des Dadaismus entlarvt werden, dessen prominenter Vertreter der bereits genannte Kurt Schwitters war.

Ein Beispiel der Anspielung auf einen konkreten Text wäre die Erwähnung von „Gofid Letterkerls Meisterwerk Zanilla und der Murch“ (Moers 2015: 38), aus dem auch ein Pseudozitat stammt:

„Ich bin nur eine Berghutze“, rief die Zanilla-Darstellerin gerade voller Dramatik, ,und du, mein Geliebter, du bist ein Murch. Wir werden niemals zueinanderfinden. Laß uns gemeinsam von der Dämonenklamm springen!“ (ebd., 38)

Die unglückliche Liebe, die in einem Selbstmord der beiden Liebenden endet, hat Gottfried Keller in der Novelle Romeo und Julia auf dem Dorfe beschrieben. Hier wird durch das Einbeziehen der Dämonenklamm - eines vermeintlichen zamonischen geografischen Phänomens - wiederum die ,zamonische“ Note in einen klassischen literarischen Text gebracht. Dies bringt wie im Fall von Nurnenwald einen humorvollen Ton in die Erzählung und aktualisiert die klassische Literatur durch den neuen phantastischen Kontext.

Anspielungen auf andere literarische Werke tauchen auch auf der Ebene der Handlung auf, so dass sie nicht als solche markiert werden. Conrad (2011: 291) macht zum Beispiel auf die Parallele zwischen dem vergifteten Buch, mit dem Pfistomefel Hildegunst betäubt, und dem vergifteten Buch aus Ecos Der Name der Rose aufmerksam. Diese Anspielungen sind schwerer erkennbar, weil sie nicht markiert und enger mit der Handlung verknüpft sind, so dass sie mit dem Kontext zusammenfließen.

Auch in Die unendliche Geschichte kann man eine Fülle an literarischen Anspielungen nachweisen, was von mehreren Autoren bemerkt worden ist. Sie enthalte z. B. ,zahlreiche Märchen-Motive, Bausteine aus der kanonisch gewordenen phantastischen Literatur der vergangenen Jahrhunderte, typische Strukturen des Bildungsromans, des Abenteuer- und Reiseromans, biblisch-religiöse Beziehungen, mythologische Bilder aus unterschiedlichen Kulturquellen, Anspielungen auf literarische Texte und auf real existierende Bilder von Edgar Ende und anderen surrealistischen Malern." (Stoyan 2004: 121) Ende verwende „nebeneinander typische Märchenmuster [...], typische Strukturen des Abenteuer- sowie Bildungsromans, psychoanalytische und anthropologische Modelle der Selbstfindung einerseits und der Initiation andererseits.“ (Lehnert 1990: 182-183) Die Anspielungen sind jedoch weniger offensichtlich als bei den anderen zwei Romanen, da sie nicht als solche markiert sind und auf der Ebene der Handlung auftreten und dadurch gänzlich mit dem Kontext zusammenfließen. Dieses Buch handelt nicht von anderen Büchern, außer dem Buch „Die unendliche Geschichte“, das Bastian liest, die Anspielungen treten also auf als Teil der Handlung und nicht etwa als Teil der Figurenrede wie in Tintenherz und sind nicht 
durch einen Kontext begleitet, der sie als Werk eines anderen Autors markiert. Als Beispiel dafür kann Atréjus Gespräch mit dem Werwolf angeführt werden. Atréju stellt sich als „Niemand“ vor und erzeugt damit eine Situation, wie sie aus der Odyssee bekannt ist, wo sich Odysseus dem Riesen Poliphem als „Niemand“ vorstellt und ihn dadurch überlistet. Das Herstellen der intertextuellen Verbindung wird völlig dem Leser überlassen, da weder in der Figuren- noch in der Erzählerrede etwas steht, was Atréjus Worte als Anspielung auf die Szene aus der Odyssee entlarven würde. Die Szene kann deshalb auf zwei Ebenen gelesen werden: Reale Lesende, denen die Odyssee nicht bekannt ist, lesen das Kapitel ungestört und fragen sich nicht, ob ihnen Information für das Verständnis der Szene fehlt. Denn für die weitere Handlung ist die Kenntnis des ursprünglichen Kontextes der Situation nicht notwendig und die Leser, die diese Information nicht haben, können nur Atréjus Tücke bewundern, ohne sich zu fragen, ob vielleicht andere Verbindungen in diesem „Niemand“ stecken. Nur die Lesenden, die über die Kenntnis über die Odyssee verfügen, können die Szene auf der zweiten Ebene lesen, indem sie das bekannte Vorwissen aktivieren und die intertextuelle Verbindung herstellen. In der Regel fehlen den kindlichen Lesern die Kenntnisse, um die intertextuellen Verbindungen zu erkennen, während sie einem Erwachsenen mit größerer Wahrscheinlichkeit zugänglich sind.

Die anderen Quellen für die Motive Phantásiens sind unter anderem die klassische Mythologie, postmoderne Literatur, Märchen (AURYN als magischer Gegenstand, der Wünsche erfüllt), die Bibel und das Christentum (Xayíde als die verführerische Frau; das Baden in den Wässern des Lebens als Wiedergeburt ähnelt der Taufe) und andere. Ende arbeitet auch mit traditionellen Symbolen, die nicht der einen oder anderen Mythologie zugeschrieben werden können. Im Bild des AURYNS verbirgt sich eine Symbolik, die sowohl der indischen als auch der griechischen Mythologie entstammen kann, wobei das Symbol „verdoppelt“ wird - aus einer Schlange, die sich selber in den Schwanz beißt und den ewigen Kreislauf des Universums oder der Zeit bedeutet (vgl. Bauer/Dümotz/ Golowin 1992: 44), werden zwei, die helle und die dunkle, die einander in den Schwanz beißen. Die Symbolik der Ewigkeit bleibt in diesem Fall erhalten und passt sich der Moral der Geschichte - der Ewigkeit des menschlichen Wiederkehrens nach Phantásien - an. Lehnert notiert auch Quellen, die nicht aus anderen literarischen Texten stammen, sondern aus der Kunstgeschichte - so sollte z. B. die Beschreibung des Elfenbeinturms eine „frappante Ähnlichkeit mit Pieter Brueghels ,Turmbau zu Babel‘ im Kunsthistorischen Museum Wien“ (Lehnert 1990: 183) aufweisen. Auch die Verbindungen zu den tradierten Bildern sind normalerweise für die erwachsenen Lesenden klar, für Kinder aber schwieriger erkennbar. „Hier wird über die Köpfe der kindlichen Leserinnen und Leser hinweg eine Komplizenschaft zwischen dem Erzähler/Autor und den erwachsenen Leserinnen und Lesern begründet, die nicht nur in der Wiedererkennbarkeit einzelner Bilder wurzelt, sondern im künstlerischen Verfahren der Intertextualität selbst.“ (ebd., 184-185) Das mögliche Lesen auf zwei Ebenen bedeutet nicht, dass einer Gruppe Rezipierender Vorinformation fehlt, sondern, dass im Text selber Mechanismen präsent sind, die die zwei verschiedenen Lesarten zulassen. 


\subsection{Funktionen der intertextuellen Bezüge}

Zitate und Anspielungen verhalten sich in den drei Romanen unterschiedlich und spielen verschiedene Rollen. Die Zitate und Anspielungen in Tintenherz werden nicht lediglich als ein Mittel auf der Ebene des Erzählens vom Erzähler eingesetzt, sondern stellen einen Teil der Handlung dar. Weiter tauchen Figuren und Gegenstände aus anderen literarischen Texten auf, die in Tintenherz erwähnt werden: Meggie liest Tinker Bell aus Peter Pan und den standhaften Zinnsoldaten aus Andersens Märchen heraus, Mo erzählt, dass er schon mehrmals einige Gegenstände herausgelesen habe (Funke 2014: 166-167). Darin unterscheidet sich Tintenherz von Die unendliche Geschichte und Die Stadt der Träumenden Bücher, da in den letzteren die Figuren und Gegenstände aus den zitierten oder angespielten Texten nicht so offensichtlich auf die Ebene der Handlung treten. Sie bekommen im neuen Kontext eine ganz andere Rolle, so dass sich auch ihre Bedeutung völlig verändert.

Die Intertextualität in Tintenherz hat zusätzlich „die Funktion die Charaktere der Figuren darzustellen. Mo, Meggie und Elinor sind sehr belesen und integrieren diverse Primärliteratur in ihre Rede, hauptsächlich, um diese mit anschaulichen Vergleichen auszuschmücken." (Heber 2010: 81) Die belehrende Moral des Textes ist, dass die positiven Charaktere eine Neigung zur Rezeption besonders von Kinder- und Jugendliteratur haben, während die Bösewichte Bücher zerstören wollen.

Die Stadt der Träumenden Bücher verzichtet durchaus auf solche didaktischen Lehren. Für diesen Roman ist die Funktion der Zitate und Anspielungen vor allem die „Erfrischung" der klassischen Werke und Autoren durch Wiederbelebung und Humor. Die Zitate und Anspielungen werden teilweise als solche markiert - am aufschlussreichsten ist dabei das Kapitel „Das Ormen“ (Moers 2015: 228-238) -, teilweise aber in die Handlung integriert und nicht durch Kontext als Intertext markiert. Die Zuschreibung der Autorschaft der klassischen Texte an phantastische Gestalten, teilweise mit lustigen Namen, und das Einsetzen der phantastischen Gestalten oder Räume in diese Texte wirken humorvoll und aktualisieren wieder die Klassiker der deutschsprachigen und europäischen Literatur. Das Entziffern der Anagramme verzögert zusätzlich den Leseprozess für die Leser und Leserinnen, die das intertextuelle Spiel erkennen. Denn ähnlich wie bei Endes Roman kann auch Moers ' Werk ruhig nur als spannende Phantastik gelesen werden, ohne dass jemand, der nicht in der Lage ist, die intertextuellen Verbindungen herzustellen, Informationen vermissen würde. Wenn jemand „Sanotthe von Rhüffel-Ostend“ lediglich als eine „halbverrückte[...] florinthische[...] Poetin“ (Moers 2015: 233) deutet, wird zwar nur die einfachere Lesart aktiviert, das Verständnis des Romans aber dadurch nicht beeinträchtigt.

In Die unendliche Geschichte sind die Anspielungen und Zitate meistens nicht als solche markiert, sondern völlig in den neuen Kontext eingebettet und eng miteinander verknüpft. Durch den neuen Kontext bekommen sie auch eine neue Rolle und Aussage: 
Das Zitat „Tu Was Du Willst“ bekommt in Die unendliche Geschichte eine neue Bedeutung, als es zur Aufforderung wird, dass Bastian seinen „Wahren Willen“ (Ende 2014: 455), seinen innerlichen Trieb finden muss, um den Weg nach Hause wiederzufinden, es wird aber nicht als Zitat markiert. Das Aufgreifen der tradierten Motive hat in Die unendliche Geschichte die Funktion, die zweite, ,gebildete“ Lesart zu öffnen. Auch trägt es stark zur Aussage des ganzen Romans bei. Das ganze Phantásien stellt die Gesamtheit menschlicher Phantasie, Schöpfungskraft und daraus stammender Literatur und Kunst dar. Es macht Sinn, dass es deshalb vorwiegend aus den literarischen und künstlerischen Motiven besteht. Wenn Bastian ins Buch steigt, steigt er zugleich in alle anderen Geschichten, die jemals existierten. Die Welt Phantásiens ist aus tradierten Motiven zusammengesetzt, weil sie alle Teil einer unendlichen Geschichte sind. Durch das Aufgreifen der bekannten Motive wird diese Aussage noch vertieft.

Gleichzeitig hat die Anwesenheit der intertextuellen Elemente dieselbe Funktion wie bei Die Stadt der Träumenden Bücher - es wird die zweite, ,gebildete“ Lesart als Möglichkeit für die Leser angeboten, die über ein genügendes Vorwissen verfügen.

\begin{tabular}{|l|l|l|l|}
\cline { 2 - 4 } \multicolumn{1}{c|}{} & $\begin{array}{l}\text { Die unendliche } \\
\text { Geschichte }\end{array}$ & Tintenherz & $\begin{array}{l}\text { Die Stadt der } \\
\text { Träumenden Bücher }\end{array}$ \\
\hline Markiertheit & $\begin{array}{l}\text { Fast immer } \\
\text { unmarkiert }\end{array}$ & Immer markiert & Teilweise markiert \\
\hline $\begin{array}{l}\text { Quellen der Zitate } \\
\text { und Anspielungen }\end{array}$ & $\begin{array}{l}\text { Märchen } \\
\text { Klassische Literatur } \\
\text { Postmoderne Literatur } \\
\text { Klassische Mythologie } \\
\text { Christliche Mythologie } \\
\text { Kunstgeschichte }\end{array}$ & $\begin{array}{l}\text { Kinder- und } \\
\text { Jugendliteratur } \\
\text { Einige Klassiker }\end{array}$ & $\begin{array}{l}\text { Klassische Literatur } \\
\text { Postmoderne Literatur }\end{array}$ \\
\hline $\begin{array}{l}\text { Funktionen und } \\
\text { Wirkung }\end{array}$ & $\begin{array}{l}\text { Betonung der } \\
\text { Aussage, dass } \\
\text { Phantásien die } \\
\text { Gesamtheit } \\
\text { menschlicher Kunst } \\
\text { (vor allem Literatur) } \\
\text { darstellt } \\
\text { Einladung zur } \\
\text { anspruchsvollen } \\
\text { Lesart }\end{array}$ & $\begin{array}{l}\text { Vorantreiben der } \\
\text { Handlung } \\
\text { Charakterisierung der } \\
\text { Figuren } \\
\text { Leseempfehlung für } \\
\text { die Leser }\end{array}$ & $\begin{array}{l}\text { Humor } \\
\text { Verzögerung des } \\
\text { Leseprozesses } \\
\text { Einladung zur } \\
\text { anspruchsvollen } \\
\text { Lesart }\end{array}$ \\
\hline
\end{tabular}

Tabelle: Intertextualität in Romanen 


\section{4 SCHLUSSFOLGERUNGEN}

Im Beitrag werden die intertextuellen Bezüge (Zitate und Anspielungen) in drei phantastischen Romanen untersucht. Den Romanen ist gemeinsam, dass sie ältere Texte zitieren oder auf diese anspielen. Diese Bezüge zu anderen literarischen Texten haben in den analysierten Romanen verschiedene Funktionen: Sie können didaktisch eingesetzt werden, zur Charakterisierung der Figuren beitragen und die Handlung bewegen, Komik erzeugen oder die Welt, in der sich die Hauptfigur bewegt, als eine literarische Welt, die vorwiegend aus bekannten Motiven zusammengesetzt ist, präsentieren.

In Die unendliche Geschichte besteht die Welt, die der Held durch seine Imagination formt, fast nur aus bekannten literarischen Motiven, sie ist sozusagen bloße Literatur, in der er sich als Mensch bewegt. Die Zitate und Anspielungen aus anderen literarischen Texten, Mythen oder auch künstlerischen Werken stützen die Idee, dass sich die menschliche Phantasie ständig erneuern muss, um eine heile außerliterarische Welt zu erhalten.

In Tintenherz werden die intertextuellen Beziehungen als Mottos zu den einzelnen Kapiteln oder als Teil der Figurenrede oder Figurengedanken eingesetzt, meistens um die Figuren zu charakterisieren; es ist zu bemerken, dass nur die positiven Figuren über Literatur sprechen und diese zitieren. Die Quellen der Bezüge sind überwiegend kinderfreundliche Texte. Dies ermöglicht die Identifikation des kindlichen Lesers mit dem Text und vor allem mit den positiven Figuren, wirkt aber auch als Leseempfehlung und ist also didaktisch eingesetzt.

Die Stadt der Träumenden Bücher legt viel Wert auf Humor und Zitate und Anspielungen darin erwecken oft Komik; sie haben eine spielerische, erheiternde Rolle. Sie sind aber auch Wegweiser für den jungen Schriftsteller, der die Hauptfigur des Romans ist die alten Klassiker haben ihm geholfen, seine schriftstellerische Karriere anzufangen.

Da die Bezüge in Die unendliche Geschichte und Die Stadt der Träumenden Bücher weniger offensichtlich markiert sind als bei Tintenherz, sind diese als komplexere Texte anzusehen. Sie eröffnen die Möglichkeit der zweifachen Lesart, die darauf basiert, dass die Leser die Bezüge als intertextuelle Phänomene erkennen, oder aber nicht, wodurch die Romane sowohl für die anspruchsvollen als auch weniger anspruchsvollen Leser rezipierbar sind.

\section{QUELLEN- UND LITERATURVERZEICHNIS}

\section{Primärliteratur}

DOLLINGER, Anja/Walter MOERS (2012): Zamonien. Entdeckungsreise durch einen phantastischen Kontinent. München: Albrecht Knaus Verlag.

ENDE, Michael (2014): Die unendliche Geschichte. Stuttgart: Thienemann-Esslinger Verlag. 
FUNKE, Cornelia (20145): Tintenherz. Hamburg: Oetinger Taschenbuch.

GOETHE, Johann Wolfgang von (1987): Sämtliche Werke. Bd. 2.1: Erstes Weimarer Jahrzehnt 1775-1786. München: Carl Hanser Verlag.

MOERS, Walter (201531): Die Stadt der Träumenden Bücher. München: Piper Verlag.

RABELAIS, François (1981): Gargantua in Pantagruel I. Ljubljana: Cankarjeva založba. SHAKESPEARE, William (2001): Twelfth Night, or, What You Will. London: Penguin Books.

\section{Sekundärliteratur}

BAUER, Wolfgang/Irmtraud DÜMOTZ/Sergius GOLOWIN (1992): Lexikon der Symbole. Mythen, Symbole und Zeichen in Kultur, Religion, Kunst und Alltag. München: Wilhelm Heyne Verlag.

BERNDT, Frauke/Lily TONGER-ERK (2013): Intertextualität. Eine Einführung (Grundlagen der Germanistik, 53). Berlin: Erich Schmidt Verlag.

CONRAD, Maren J. (2011): Von toten Autoren und Lebenden Büchern. Allegorien und Parodien poststrukturalistischer Literaturtheorie in den Katakomben der Stadt der Träumenden Bücher. In: G. Lembke (Hrsg.): Walter Moers' Zamonien-Romane. Vermessungen eines fiktionalen Kontinents. Göttingen: V\&R unipress, 281-302.

GENETTE, Gérard (1993): Palimpseste. Die Literatur auf zweiter Stufe. Frankfurt a. M.: Suhrkamp.

HEBER, Saskia (2010): Das Buch im Buch. Selbstreferenz, Intertextualität und Mythenadaptation in Cornelia Funkes Tinten-Trilogie. Kiel: Verlag Ludwig.

JUVAN, Marko (2000): Intertekstualnost (Literarni leksikon. Študije. Zv. 45). Ljubljana: DZS.

LEHNERT, Lehnert (1990): Moderne und postmoderne Elemente in der „phantastischen“ Kinderliteratur des 20. Jahrhunderts. In: H.-H. Ewers, M. Lypp, U. Nassen (Hrsg.): Kinderliteratur und Moderne. Ästhetische Herausforderungen für die Kinderliteratur im 20. Jahrhundert. Weinheim/München: Juventa Verlag, 175-195.

MADER, Ilona (2012): Metafiktionale Elemente in Walter Moers' Zamonien-Romanen (Innsbrucker Studien zu Literatur und Film der Gegenwart, Band 1). Marburg: Tectum Verlag.

NÜNNING, Ansgar (Hrsg.) (2013): Metzler Lexikon Literatur- und Kulturtheorie. Ansätze-Personen - Grundbegriffe. 5. Auflage. Stuttgart: J. B. Metzler Verlag.

STOYAN, Hajna (2004): Die phantastischen Kinderbücher von Michael Ende. Frankfurt a. M.: Peter Lang. 


\section{POVZETEK}

\section{Medbesedilnost pri Michaelu Endeju, Cornelii Funke in Walterju Moersu}

Članek obravnava medbesedilne navezave v romanih Neskončna zgodba (Die unendliche Geschichte), Srce iz črnila (Tintenherz) in Mesto sanjajočih knjig (Die Stadt der Träumenden Bücher). Analizirani so citati in namigi v posameznih romanih; njihove funkcije segajo od razkrivanja literarnega sveta kot skupka samih citatov, karakterizacije figur in vzgoje mladih bralcev do humorja.

Ključne besede: medbesedilnost, citiranje, Neskončna zgodba, Srce iz črnila, Mesto sanjajočih knjig

\section{ABSTRACT}

\section{Intertextuality in the Works of Michael Ende, Cornelia Funke and Walter Moers}

This paper discusses intertextual phenomena in the novels The Neverending Story, Inkheart, and The City of Dreaming Books. Quotations and references in the novels are analysed as well as their functions, which range from showing the literary world as a net of quotations, characterisations of personas, and developing a sense of humour in young readers.

Key words: intertextuality, quotations, The Neverending Story, Inkheart, The City of Dreaming Books 